Radiation Carcinogenesis at Low Doses

Abstract. An analysis of experimental findings indicates that the induction of $a$ mammary neoplasm in the Sprague-Dawley rat is dependent on the action of radiation on more than one cell. Although a linear relation between incidence and $x$-ray dose might be consistent with available data, such a relation would be fortuitous and linear extrapolation to lower doses is unjustified.

Carcinogenesis induced at low radiation doses is a problem of crucial importance to modern radiation technology. Organizations responsible for recommending values of the maximum permissible dose (MPD), such as the International Commission on Radiological Protection and the National Council on Radiation Protection and Measurements, have stated that "prudence" requires the assumption that the frequency of harmful effects is proportional to dose at low doses (1). Although these organizations have made it plain that this assumption is intended to provide a conservative estimate of the upper limits of incidence (2), calculations based on this assumption have been performed to predict that there would be a large number of cancers induced in the population of the United States each year if it were to be exposed near the MPD levels (3). Bond has pointed out that exposure near the MPD of even a large fraction of the population is virtually impossible and also that the incidence frequencies employed in these calculations are probably many times too large (4). These factors might reduce the calculated incidence by 100 -fold or more, but adherence to linear extrapolation indicates nevertheless a finite incidence of cancer at all doses. This reasoning would also imply the need for rigorous control of the exposure of individuals to radiation for medical purposes and might even induce attempts to minimize exposure to natural background radiation which would represent the principal source of radiation carcinogenesis in the population.

It has not been possible thus far to check the validity of the "prudent assumption" of linearity between dose and effect by direct observation at absorbed doses of x-rays of less than several rads. However, we present here some pertinent conclusions that can be drawn on the basis of data obtained at higher doses in cases where radiations of high linear energy transfer (neutrons) have been experimentally applied. Such conclusions will be presented here. Although the analysis does not provide a definitive answer to the basic question of whether there exists a "threshold dose" for cancer induction, it may serve to clarify some of the issues involved.

The absorbed dose is delivered by individual charged particles, that is, in discrete statistically independent events of energy deposition. The spectrum of energy deposition events is independent of the magnitude of the dose. From this fact one can conclude (5) that in the limit of very low doses any effect is proportional to dose if it can be produced by a single particle. If $n$ particles are involved in the induction of the effect, the dose-effect relation must be proportional to the $n$th power of the dose when the dose is small (that is, if the frequency of particles in the volume of interest is much less than unity). Even if $n$ is only 2, a linear downward extrapolation by a factor of 10 will, under these conditions, introduce an overestimate by a factor of 10 since the incidence, instead of being 10 times less, will in fact be 100 times less.

The question of whether single particles are involved in the production of neoplasms can be analyzed in the case of mammary neoplasms of the SpragueDawley rat. Like most other malignancies of interest in radiation research, these tumors are atypical since they occur with appreciable frequency in control animals. In addition, less than half

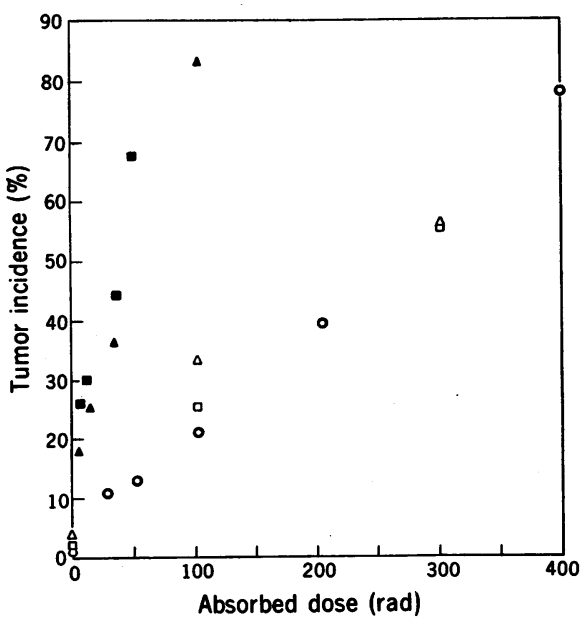

Fig. 1. Percentage of Sprague-Dawley rats with mammary neoplasms induced by various doses of $\mathrm{x}$-rays and neutrons [x-rays: $O$, Bond et al. (6); $\triangle$, Shellabarger (8); $\square$, Vogel and Zaldivar (9); neutrons: $\boldsymbol{\Delta}$, Shellabarger $(8) ; \square$, Vogel $(7)]$. of these tumors are malignant. Nevertheless, these data have been used to obtain estimates of cancer incidence at low doses, and there are various aspects of the following discussion that should apply to carcinogenesis in general.

Figure 1 shows data by various authors (6-9) on the incidence of neoplasms as a function of the dose of x-rays (200- to 250-kv energy) and "fission" neutrons (10). The following discussion is concerned with the mechanism of tumor induction at low doses. The data points in the range of high doses where tumor incidence reaches a maximum value and subsequently declines $(6,9)$ are therefore not included in Fig. 1. The various studies appear to be in substantial agreement, and, in the case of x-rays, a linear dependence of incidence on dose appears to be consistent with the data, but linearity seems less consistent with the results for neutrons.

This conclusion becomes much more evident when the data points are plotted on a logarithmic representation where linearity should result in lines with a slope of 1 . Such a representation is shown in Fig. 2, where the mean number of tumors induced per animal is plotted instead of the fraction of animals with tumors. The two quantities coincide at small incidences; at larger doses the number of tumors per animal has been taken from the original work (6) or, where not observed experimentally (7-9), has been deduced from the fraction of animals with tumors. The correction for multiple tumors has been based on the assumption that the tumors occur independently (see below). The spontaneous incidence has been subtracted. Neither set of data appears to be consistent with linearity. However, a logarithmic plot of the ratio of doses for equal incidence (the relative biological effectiveness) versus the $\mathrm{X}$-ray dose approximates lines with slopes of -1 at low doses (Fig. 3). This is in accord with previous observations (5) and with the theory of dual radiation injury (11).

According to this theory, lesions produced by a single neutron secondary particle (usually a proton) require the action of two $x$-ray secondary particles (electrons). Hence in the range covered in Fig. 3 the primary lesions underlying the effect must have predominantly a quadratic dependence on $\mathrm{x}$-ray dose. This does not eliminate the possible existence of a weak linear component for radiation of low linear energy transfer that might become 
dominant at $\mathrm{x}$-ray doses that are much lower than those covered in Figs. 1-3. It must, however, be concluded that, even if the dose-effect relation for X-rays were in fact linear in the dose range investigated (a distinct possibility in view of the limited accuracy of the experiments), the linearity must be accidental and there is no justification for a linear extrapolation.

The complexity of the process is apparent because there is no linear relation for neutrons, since at low doses the slope in the logarithmic representation is less than 45 degrees. Indeed, at first sight, it would appear that the assumption of linearity is not conservative but instead underestimates the hazard at low doses since the incidence increases with a power of the neutron dose that is less than unity.

Microdosimetric determinations (12) have shown that it requires a dose of somewhat over 20 rads of fission neutrons to achieve an average of one traversal through cell nuclei having a diameter of about $7 \mu \mathrm{m}$. The neutron dose-effect curve extends well below this radiation dose, and near its lower range the probability of one neutron secondary particle per nucleus and even per cell approaches 0.1. This result, however, does not imply that individual cells are the foci of carcinogenesis. On the contrary, the fact that the dose-effect relation deviates from linearity at a dose where the mean number of neutron secondary particles in a cell is considerably less than unity implies that the development of malignancies must be determined by radiation effects on a number of interacting cells. It may be helpful if some explanatory remarks precede the formal proof of this assertion.

It has been generally observed that biological variability of radiosensitivity flattens the dose-effect curve; whenever the more sensitive subgroups of the population are exhausted by nearly full incidence, the logarithmic slope of the dose-effect relation decreases. One might therefore assume that variance of sensitivity between animals or between cells in individual animals may explain the observed dose-effect relations.

An analysis of the frequency of multiple tumors (6) shows that this frequency follows Poissonian statistics, which eliminates the possibility that the incidence of one tumor decreases the induction probability of further malignancies in the animal. It is therefore possible to correct the original data which represent the fraction of animals having at least one tumor and to derive

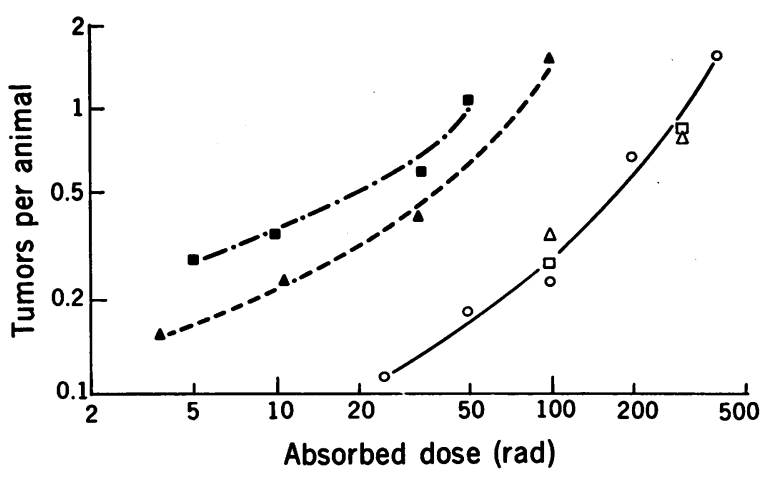

Fig. 2. Mean number of tumors per animal as a function of $x$-ray and neutron dose. The data correspond to those given in Fig. 1, but they are corrected for multiple tumor incidence and spontaneous incidence is subtracted [x-rays: $\mathrm{O}$, Bond et al. (6); $\triangle$, Shellabarger $(8)$; $\square$, Vogel and Zaldivar (9); neutrons: $\Delta$, Shellabarger $(8) ; \square$, Vogel (7)].

the total number of tumors produced. This correction has been applied to Fig. 2 , in which saturation can therefore not be a factor. Variations in sensitivity between different cells cannot be invoked because an incidence that leads to saturation of a sensitive subgroup cannot occur at doses where the event frequency in a cell is much lower than unity.

Biological variability alone therefore cannot explain the observed results. If, on the other hand, the combined effect of two or more neutron secondary particles caused the carcinogenetic transformation, the slope of the dose-effect curve would have to be greater, rather than less, than 1. There remains the possibility that the transformation is initiated by one particle, but that a second particle kills the cell. It has been suggested that this kind of process occurs at higher doses of the order of 100 rads, where the level of effect reaches a maximum with subsequent decline, but it also cannot operate under conditions where the probability of two events is much less than the probability of one event.

We now present the formal proof for the fact that the observed frequency of tumor incidence cannot be the direct reflection of the frequency of carcinogenetic transformation in individual cells. The theorem to be proved is the following: Assume that carcinogenesis is due to alteration of one cell in some population of cells which may have diverse radiosensitivity, but which do not interact. Then in a double-logarithmic plot the slope of the dose-effect relation must at least be equal to $(1-n)$, where $n$ is the mean number of charged particles traversing a cell. When $n$ is small as compared to 1 , the slope of the dose-effect curve cannot be significantly less than 1 .

To prove this statement one must start from the most general assumption concerning the dose-effect relation for the cells. The probability that the effect takes place when a cell selected at random from the population is subjected to exactly $v$ events of energy deposition will be called $E_{v}$, and it must be assumed that these probabilities can have any values between 0 and 1 . The dose-effect relation is then the result of the multiplication of these values by their relative frequencies, which are determined by Poissonian statistics:

$$
E(D)=\sum_{\nu=1}^{\infty} E_{\nu} e^{-\phi D} \frac{(\phi D)^{\nu}}{v !}
$$

where $n=\varnothing D$ is the mean number of charged particle traversals at dose $D$, that is, the expectation value of the event number $v$.

From this general formula one can deduce without further assumption that the slope in the double-logarithmic representation must always be at least equal to $(1-\varnothing D)$. This result is derived as follows:

$$
\begin{aligned}
& \frac{d \ln E(D)}{d \ln D}=\frac{D}{E(D)} \frac{d E(D)}{d D}= \\
& \frac{D}{E(D)} \sum_{\nu=1}^{\infty} E_{\nu}\left[e^{-\phi D} \frac{(\phi D)^{\nu-1}}{(\nu-1) !} \phi-\phi e^{-\phi D} \frac{(\phi D)^{\nu}}{\nu !}\right]
\end{aligned}
$$

By use of Eq. 1 and a rearrangement of terms one obtains:

$$
\frac{d \ln E(D)}{d \ln D}=\frac{\sum_{\nu=1}^{\infty} E_{\nu} e^{-\phi D} \frac{(\phi D)^{\nu}}{v !}(v-\phi D)}{\sum_{\nu=1}^{\infty} E_{\nu} e^{-\phi D} \frac{(\phi D)^{\nu}}{v !}}
$$

or

$$
\begin{aligned}
& \frac{d \ln E(D)}{d \ln D}=
\end{aligned}
$$

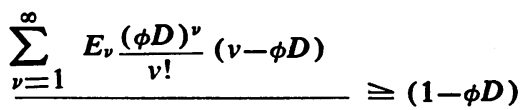

$$
\begin{aligned}
& \sum_{\nu=1}^{\infty} E_{\nu} \frac{(\phi D)^{\nu}}{v !}
\end{aligned}
$$




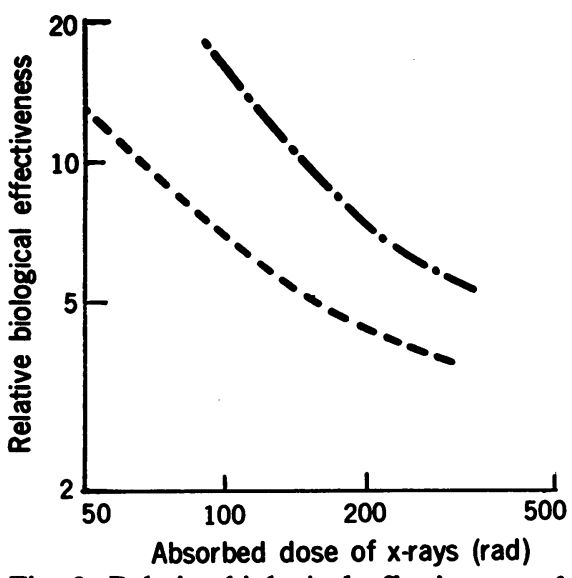

Fig. 3. Relative biological effectiveness of neutrons versus $x$-rays as a function of $x$-ray dose. The curves are derived from the interpolated lines in Fig. 2 [(-...-) Shellabarger (8); (_. . . . Vogel (7)].

The conclusion that the slope is greater than or equal to $(1-\phi D)$ follows from the fact that the numerator in Eq. 4 , contains the same positive terms as the denominator, each term being multiplied by the factor $(\nu-\phi D)$ which is always at least as large as $(1-\varnothing D)$. This result proves the theorem.

As stated above, $\varnothing D$ is of the order of 0.1 at the lowest doses of fission neutrons. The assumption of unicellular origin for the malignancy then requires the slope of the logarithmic dose-effect line to be no less than 0.9. Since the slope is, in fact, 0.5 or less, it must be concluded that in the dose range investigated the carcinogenetic process cannot reflect radiation injury to individual cells in a population of noninteracting cells. This statement applies even if these cells have an arbitrary distribution of sensitivities and if the "carcinogenesis" consists in the induction of a variety of neoplasms.

The process must therefore involve energy absorption by more than one cell, and the situation is too complicated to warrant a linear extrapolation to low doses. To account for the shallow slope in the region investigated, it is necessary to assume that radiation absorption events can both promote and inhibit tumor induction which thus must involve more than one kind of process as well as more than one cell.

As mentioned earlier, the frequency of multiple tumors (6) appears to follow a Poissonian distribution; in particular, the maximum fraction of animals with only one tumor is very close to 37 percent, as would be expected if there are no interactions between tumors. The inhibitory effect deduced can therefore not be one exerted by the presence of other tumors. The carcinogenetic action seems, in fact, to be controlled by local phenomena, and the process has been termed "scopal," not only because tumors were induced only in the portion of the animal irradiated but also because they have been induced with apparently equal efficiency when small pieces of mammary tissue were irradiated in vitro and then reimplanted (13). Although hormonal and immunological factors controlling tumor development have been identified when whole animals are exposed (14), there seems to be no known evidence for local radiation-induced inhibitory action.

With the complexity of the tumor induction process established, there remains little justification for linear extrapolations, and this conclusion, in turn, removes apparent inconsistencies between the dose-effect relation and postulates (15) and histological evidence (16) to the effect that carcinogenesis requires the transformation of several contiguous cells. There is, however, at present, insufficient evidence for numerical estimations of tumor incidence based on linear or other extrapolations.

HARALD H. ROSSI

Albrecht M. Kellerer

Radiological Research Laboratories, Department of Radiology, College of Physicians and Surgeons of Columbia University, New York 10032
1. Recommendations of the International Commission on Radiological Protection (International Commission on Radiological Protection Publication No. 9, Pergamon, New York, 1966); Basic Radiation Protection Criteria (Report No. 39, National Council on Radiation Protection and Measurements, Bethesda, Md., 1971), p. 58.

2. International Commission on Radiological Protection, Health Phys. 12, 302 (1966).

3. J. W. Gofman and A. R. Tamplin, testimony presented before the Subcommittee on Air and Water Pollution, Committee on Public Works, U.S. Senate, 91st Congress, 18 November 1969.

4. V. P. Bond, paper presented at the Council for the Advancement of Science Writers, 8th Annual Briefing on New Horizons in Science, Raleigh, N.C., 16 November 1969.

5. H. H. Rossi, Phys. Med. Biol. 15 (No. 2), 255 (1970).

6. V. P. Bond, E. P. Cronkite, S. W. Lippincott, C. J. Shellabarger, Radiat. Res, 12, 276 (1960).

7. H. H. Vogel, Nature 222, 1279 (1969)

8. C. J. Shellabarger, unpublished data.

9. H. H. Vogel and R. Zaldivar, paper presented at the Atomic Energy Commission symposium on Neutrons in Radiobiology, Oak Ridge, Tenn., CONF-691106 (1969).

10. In view of the limited accuracy of these data, the slight numerical difference between exposure (in roentgens) and absorbed dose (in rads) has been ignored. All data have been obtained or derived for tumors appearing within 11 months after exposure to radiation.

11. A. M. Kellerer and H. H. Rossi, Radiat: Res. 47, 15 (1971).

12. H. H. Rossi, Adian. Biol. Med. Phys. 11, 27 (1967).

13. C. J. Shellabarger and R. W. Schmidt, Nature 218, 192 (1968)

14. K. Yokoro, J. Furth, N. Haran-Ghera, Cancer Res. 21, 178 (1961).

15. G. Failla, Radiology 69, 23 (1957).

16. M. C. Rigden, D. W. Brigham, J. S. Nelson, F. B. Hershey, Surg. Forum 25, 356 (1964).

17. We thank Dr. V. P. Bond for many productive discussions. Work performed under U.S. Atomic Energy Commission contract AT-(301)-2740.

18 June 1971; revised 17 August 1971

\section{Morphological Transformation in vitro of Human Fibroblasts}

\section{by Epstein-Barr Virus: Preliminary Observations}

Abstract. Human embryo fibroblasts have undergone morphological transformation in vitro after infection by Epstein-Barr virus. The fibroblasts were maintained in suspension during exposure to the virus, and further treatment with inactivated Sendai virus increased the transformation rate. The transformed cells were large and polygonal and grew in discrete, heaped up, foci.

Since the first discovery of EpsteinBarr (EB) virus in cultured Burkitt lymphoma cells (1), evidence implicating this agent as a possible cause of the tumor has steadily grown. Thus, the virus stimulates human lympho-proliferation both in vitro (2) and in vivo (3) and is linked with the Burkitt lymphoma both on seroepidemiological grounds (4) and because the tumor cells carry virus-determined surface neoantigens (5) as well as the viral genome (6).

With a suspected human tumor virus, there are great difficulties in devising experiments to show conclusively that the suspect virus in fact plays an etiological role in a particular malignant disease. Accordingly, it was considered that at the experimental level new information on the oncogenic potential of EB virus might be obtained if some conventional demonstration of in vitro cellular transformation could be achieved. Although it has long been known that EB virus cannot be made to infect any of a wide variety of monolayer test tissue cultures by standard techniques (7), it was thought that some special manipulation might allow infection to take place. Experiments have therefore been 